\title{
Correction to: Mode of initial renal replacement therapy and transplant outcomes in the chronic kidney disease in children (CKiD) study
}

Meredith A. Atkinson ${ }^{1}$ • Jennifer L. Roem ${ }^{2} \cdot$ Anuradha Gajjar $^{3}$ - Bradley A. Warady ${ }^{4}$. Susan L. Furth ${ }^{5}$. Alvaro Muñoz ${ }^{2}$

Published online: 2 March 2020

(C) IPNA 2020

\section{Correction to: Pediatr Nephrol}

https://doi.org/10.1007/s00467-019-04416-2

The original version of this article unfortunately contained a mistake. Figures 1 and 2 contained undesired lines as a result of reproduction. The corrected figures are presented below.

The online version of the original article can be found at https://doi.org/ 10.1007/s00467-019-04416-2

Meredith A. Atkinson

matkins3@jhmi.edu

1 Division of Pediatric Nephrology, Johns Hopkins University School of Medicine, 200 N. Wolfe St., 3rd Floor, Baltimore, MD 21287 , USA

2 Johns Hopkins Bloomberg School of Public Health, Baltimore, MD, USA

3 Weill Cornell Medical College, New York, NY, USA

4 Children's Mercy Hospital, Kansas City, MO, USA

5 Children's Hospital of Philadelphia, Philadelphia, PA, USA 


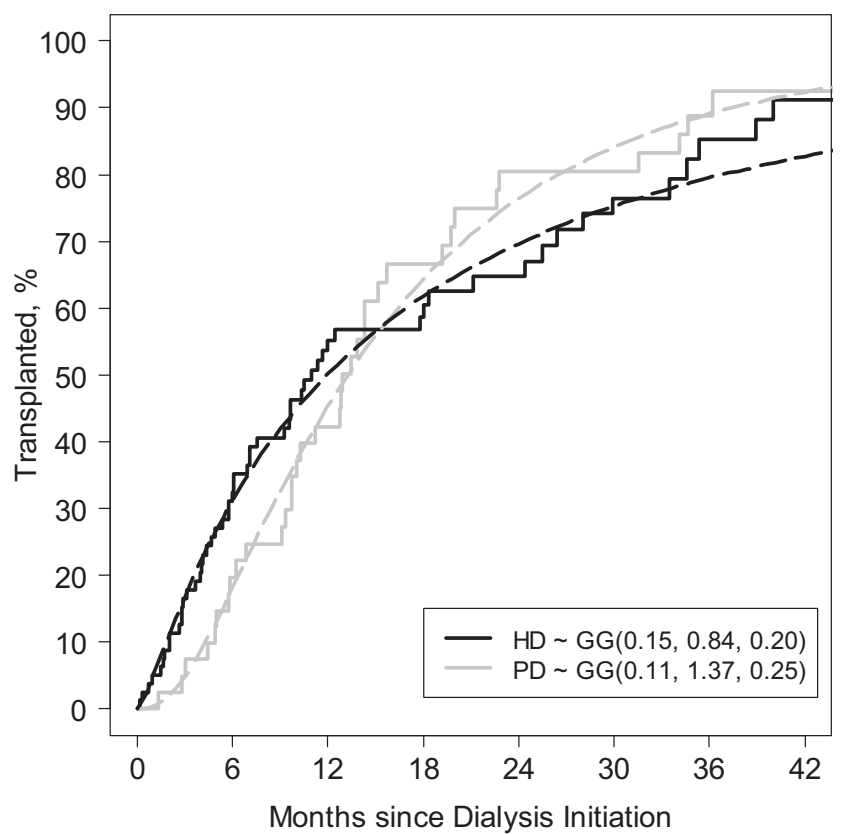

Fig. 1 Cumulative incidence of transplantation (solid lines) following dialysis as first RRT event in 123 CKiD subjects. The black lines represent the $81 \mathrm{HD}$ participants of whom $50 \%$ were transplanted by 11 months after initiating dialysis. The gray lines are the 42 PD participants of whom $50 \%$ were transplanted 13 months after initiating dialysis. The dashed lines represent the generalized gamma curves yielding a likelihood ratio statistic with a $p$ value $=0.028$

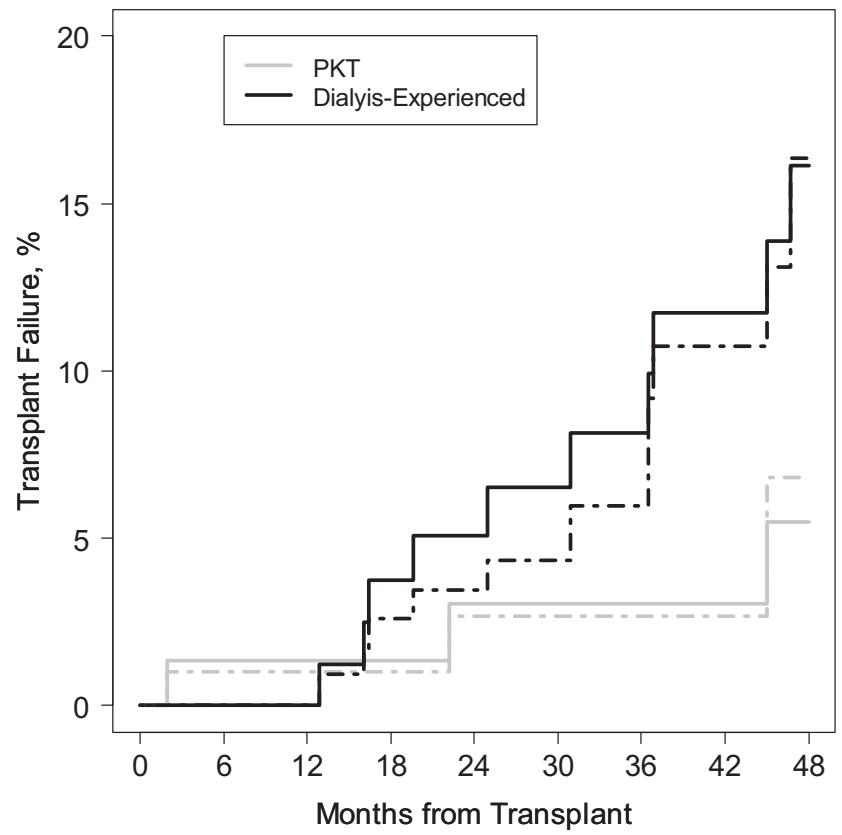

Fig. 2 Cumulative incidence of transplant failure following transplant event in 170 CKiD subjects. Solid lines depict unadjusted functions and dash-dotted lines represent adjusted functions using inverse probability weights. The gray lines are the 79 PKT participants of whom only $5 \%$ had transplant failure by 48 months. The black lines represent the 91 dialysisexperienced participants of whom $16 \%$ had transplant failure by 48 months. Incidence of transplant failure marginally differs between dialysis-experienced and PKT when unadjusted $(p=0.092)$, but attenuates when adjusted by inverse probability weights $(p=0.206)$ 